\title{
Astrometry of star-forming regions in the Sagittarius spiral arm
}

\author{
K. L. J. Rygl* \\ Italian ALMA Regional Centre, INAF-Istituto di Radioastronomia, Via P. Gobetti 101, 40129, \\ Bologna, Italy \\ E-mail: kazi.rygleinaf.it
}

\section{Y. W. Wu}

National Time Service Center, Key Laboratory of Precise Positioning and Timing Technology, Chinese Academy of Sciences, Xi'an 710600, China

E-mail: yuanwei.wuentsc.ac.cn

\section{J. Reid}

Harvard-Smithsonian Center for Astrophysics, 60 Garden Street, Cambridge, MA 02138, USA

E-mail: mreidecfa.harvard.edu

\section{A. Brunthaler, K. M. Menten}

Max-Planck-Institut für Radioastronomie, Auf dem Hügel 69, 53121 Bonn, Germany

E-mail: brunthal@mpifr-bonn.mpg.de, kmenten@mpifr-bonn.mpg.de

\begin{abstract}
We are now outlining the basic spiral structure of our Galaxy. This is not a trivial matter due to our location within the Galactic disk that causes any spiral structure to be superimposed within the line of sight. While radial velocity information can largely disentangle the various spiral arms, kinematic distances are not accurate enough for precise spiral structure studies. We measure the parallaxes and proper motions of water and $6.7 \mathrm{GHz}$ methanol masers associated with starforming regions in the Sagittarius spiral arm, up to the distance of the tangential point of $5.4 \mathrm{kpc}$ at a Galactic longitude of $49^{\circ}$, as part of the BeSSeL survey program. By combining these new astrometric data with previous measurements, we can study the distribution of high-mass starforming regions in this spiral arm. As the Sagittarius arm is one of the closest spiral arms to the Sun, the fractional parallax uncertainties are relatively small, permitting one to trace the three dimensional structure and velocities of the star forming regions in the arm to ultimately compare these to spiral arm models and observations of other (face-on) galaxies.
\end{abstract}

14th European VLBI Network Symposium \& Users Meeting (EVN 2018)

8-11 October 2018

Granada, Spain

* Speaker. 


\section{Introduction}

The Bar and Spiral Structure Legacy Survey (BeSSeL, Brunthaler et al. 2011; Reid et al. 2014) aims to measure the parallaxes and proper motions of $\sim 200$ high-mass star-forming regions (HMSFRs) in our Milky Way to study the spiral structure and its kinematics. Without accurate distance and proper motion measurements it is impossible to confidentially disentangle the various structures and velocity signatures of the spiral arms since they are superimposed in our line of sight due to our position in the Galactic plane. In other face-on galaxies, large-scale spiral arm patterns can be studied much more easily. However, due to a much superior linear resolution and sensitivity, small-scale structure of spiral arms can be much better studied in the Milky Way, provided that the astrometry is available.

The Sagittarius (Sgr) arm, as seen in the direction of the Galactic Centre, is the nearest spiral arm to the Sun. Its proximity and thus relatively small fractional astrometric uncertainties make the Sgr Near spiral arm particularly interesting to study the small-scale structures in the spiral arm and the kinematical signatures of the HMSFRs it contains. The Sgr Near spiral arm, defined as the Sgr spiral arm up to the tangential point of $5.4 \mathrm{kpc}$ distance at $l \sim 49^{\circ}$, has been traced by previous VLBI maser astrometry - 12 high-mass star-forming regions were reported on by Wu et al. (2014) and also Zhang et al. (2009a); Oh et al. (2010); Sato et al. (2010a,b); Xu et al. (2011); Kurayama et al. (2011); Chibueze et al. (2014, 2016). With the BeSSeL survey we add the astrometry of seven new HMSFRs (Rygl et al. 2019, in prep.). In these proceedings we discuss the Sgr Near spiral arm structure and velocity field based on the combined data.

\section{New maser astrometry of the Sagittarius spiral arm}

Our BeSSeL observations were carried out with the National Radio Astronomy Observatory's ${ }^{1}$ Very Long Baseline Array (VLBA). Six targets were $6.7 \mathrm{GHz}$ methanol masers, observed in four epochs, and one was a water maser, observed in six epochs. The observational strategy included geodetic-like blocks executed every 1.5-2 $\mathrm{h}$ to remove the residual multi-band delay at each antenna caused by tropospheric zenith-delay, clock offsets and drift rates. The masers were observed together with two to four position reference sources (quasars) in reverse phase-referencing mode, to calibrate both the maser and position reference sources on the strongest and most compact maser channel.

The relative position offsets between the maser and a position reference source were used to fit a sinusoidal parallax and linear proper motion. However, for the $6.7 \mathrm{GHz}$ methanol masers, the parallax values appeared to depend on the separation between the maser and the position reference, resulting in separation-dependent "parallax gradients". Reid et al. (2017) found that the probable culprit was an uncompensated ionospheric delay, and that the methanol maser astrometry that used the minimal required number of epochs for a parallax measurement (four) was particularly sensitive to this. The proposed solution was to create position data relative to an artificial quasar at the maser position, based on the relative offsets of all the available position reference sources, and perform the parallax and proper motion analysis on that (Reid et al. 2017; Wu et al. 2019). This is the

\footnotetext{
${ }^{1}$ The National Radio Astronomy Observatory is a facility of the National Science Foundation operated under cooperative agreement by Associated Universities, Inc.
} 
strategy that we followed for the sources where the position reference distribution allowed it. If not, the fitting was done as described in Reid et al. (2009a) and parallax uncertainties were inflated appropriately to include the estimated effect of the ionospheric delay (Reid et al. 2017).

\section{Sagittarius Arm structure}

While generally spiral arm memberships can be assigned based on the position-velocity information, some sources can have deviating LSR velocities due to, for example, a supernova kick. We therefore use additionally the Bayesian distance estimator of Reid et al. (2016) which considers $\left(l, b, v_{\mathrm{LSR}}, \pi\right)$ to estimate a distance probability density function and which has been improved to additionally include proper motions (Reid et al. 2019, in prep.).

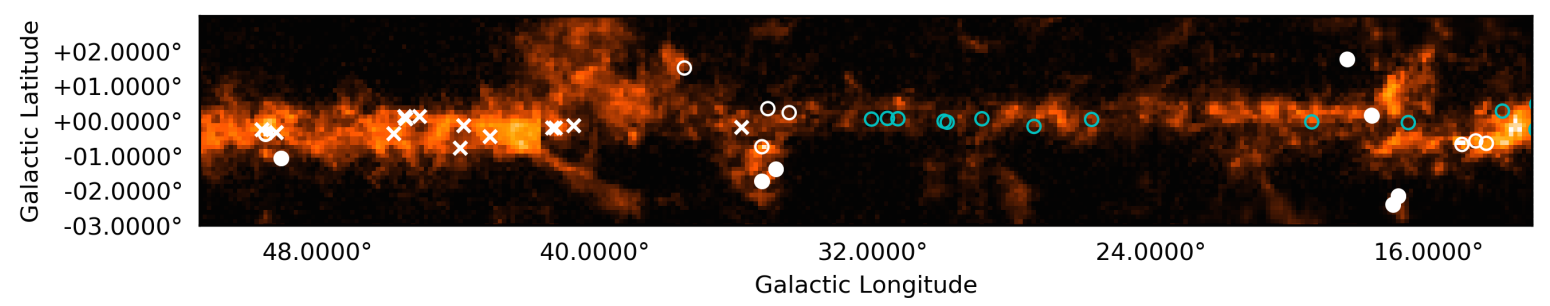

Figure 1: $C O(1-0)$ emission (Dame et al. 2001) integrated $\pm 10 \mathrm{~km} \mathrm{~s}^{-1}$ around the LSR velocity of the Sgr Near spiral arm expected from Galactic rotation. White symbols indicate HMSFRs in the Sgr arm: filled circles- this work; empty circles - Sgr Near literature values (see text for references); crosses - Sgr Far values (Wu et al. 2019), Cyan circles Scutum HMSFRs (Sato et al. 2014).

To inspect the spiral arm structure in the Galactic plane $(l, b)$ we compare in Fig. 1 the sources from the Sgr Near segment to those in the Sgr Far arm (Wu et al. 2019) and the Scutum spiral arm (Sato et al. 2014), the arm behind Sgr and thus farther away from the Sun. In addition, to point out the locations of molecular gas, we plot in Fig. 1 also the $\mathrm{CO}(1-0)$ line emission, integrated $\pm 10 \mathrm{~km} \mathrm{~s}^{-1}$ around the expected Sgr Near LSR velocity based on Galactic rotation. While the Sgr Far and Scutum maser sources all lie within $\pm 0.7^{\circ}$ of the Galactic plane, the Sgr Near sources have clearly a wider spread in latitude due to their closer distances. In particular, one can see that much of the $\mathrm{CO}(1-0)$ emission at $|b|>1^{\circ}$ has Sgr Near SFRs associated with it. The inhomogeneity of the $\mathrm{CO}(1-0)$ line emission at $|b|>1^{\circ}$ and the lack of bright maser-bearing HMSFRs at Galactic longitudes of $20-30^{\circ}$, indicate that the Sgr Near spiral arm is not a smooth structure, but an inhomogeneous collection of molecular clouds such as can be seen also in other face-on galaxies. For example, in the barred galaxy M 83 (Fig. 2), one can see the spiral arms traced by dark lanes, caused by dust extinction thus indicating the presence of high-density molecular clouds, and bright, reddened light from young early-type stars. Within the large-scale pattern of spiral arms, there is plenty of sub-structure present in the shape of feather-like features, gaps of extinction, and hubs of extinction and young stars.

Using the astrometry, we can obtain the 3D positions of the HMSFRs and examine them in a planar and in-plane view of the Galaxy (see Fig. 3). This confirms what is seen in the 2-dimensional $\mathrm{CO}(1-0)$ line integrated maps: the Sgr Near spiral is clearly structured. Most HMSFRs lie within the Sgr spiral arm width of 260 pc (Reid et al. 2014) when considering their parallax uncertainty. 


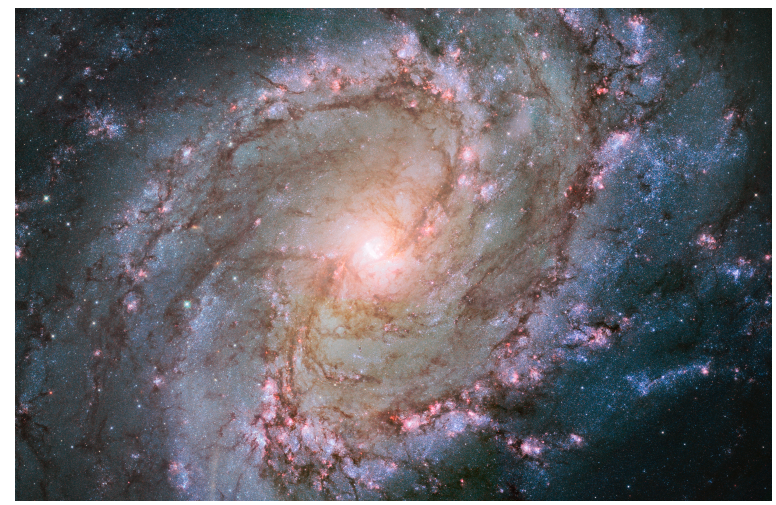

Figure 2: Hubble image of the barred galaxy M83 where the spiral arms are traced by extinction features and star-forming regions and young stars reddened by the dust. Image credits: NASA, ESA, and the Hubble Heritage Team (STScI/AURA), W. Blair (STScI/Johns Hopkins University) and R. O’Connell (University of Virginia).
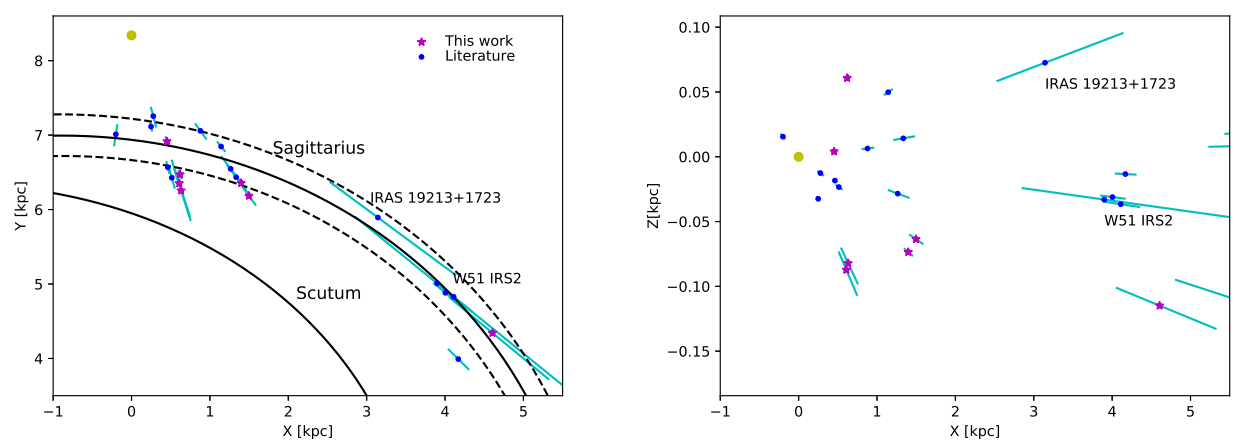

Figure 3: Left and right: VLBI maser astrometry of HMSFRs in the Sagittarius Near spiral arm on the Galactic plane, seen from above (left) and from within the plane (right). Magenta stars mark our newly measured HMSFRs, blue dots represent sources discussed in the literature (see text for references). The yellow dot at $(8.34,0)$ is the Sun. The two HMSFR with parallax uncertainties larger than $20 \%$ are indicated. A segment of the Sgr arm's log-periodic spiral (Wu et al. 2014) is drawn in solid lines. Dashed lines show the $1 \sigma$ width of the Sgr spiral arm of $0.26 \mathrm{kpc}$ (Reid et al. 2014).

Projecting the HMSFRs within the plane, shows that what might appear aligned in $\mathrm{XY}$ is not necessarily also so in $\mathrm{XZ}$ and that any structure has to be considered in three dimensions.

\section{Sagittarius Arm velocity field}

Perhaps the most interesting and new results of the BeSSeL astrometry of the Sgr Near spiral arm are the kinematical properties of HMSFRs. With the proper motions, LSR velocity, and 3D position we obtain the peculiar velocity in a co-rotating polar coordinate frame, where $U$ is the radial motion towards the Galactic Centre, $V$ is the azimuthal motion in the direction of Galactic rotation (clockwise), and $W$ is the upward motion towards the Galactic north pole. For the transformation we have used $8.34 \mathrm{kpc}$, as distance to the Galactic centre and a Galactic rotation speed of $240 \mathrm{~km}$ $\mathrm{s}^{-1}$ (Reid et al. 2014) together with the Solar motion values of $\left(U_{\odot}, V_{\odot}, W_{\odot}\right)=(11.1,12.2,7.2) \mathrm{km}$ 
$\mathrm{s}^{-1}$ (Schönrich et al. 2010). We investigate the ensemble of the literature and our preliminary $U$ and $V$ peculiar motions with respect to Galacto-centric radius and Galactic azimuth. Note that we have removed the kinematic outlier G049.04-01.07, since it has a large non-random motion, and IRAS $19213+1723$ and W51 IRS2 which had a parallax uncertainty larger than $20 \%$.

While no particular correlation is found for Galactic azimuth angle, both $U$ and $V$ tend to increase with Galacto-centric radius (Fig. 4): their correlation coefficients are 0.58 and 0.68 , respectively, indicating that the possibility for this distribution being accidental is less than 5 and 1 percent, respectively. Because for a log-periodic spiral the radius and azimuth are mathematically correlated we calculate the separation from the spiral arm centre (defined by the log-periodic of $\mathrm{Wu}$ et al. 2014) for all the HMSFRs, where HMSFRs with negative separations are inward of the arm centre, and those with positive separation are outward of arm centre. Figure 5 shows that while $V$ is still, albeit weaker, correlated with separation from spiral arm centre (chance probability $<0.05$ ), the correlation for $U$ is quite weak. The HMSFR velocity field thus suggests a kinematic pattern for the Sgr near spiral arm: inward of the spiral arm centre HMSFRs tend to rotate slower and outward of arm centre HMSFRs tend to rotate faster. Patterns for radial motion would require more and more precise data.
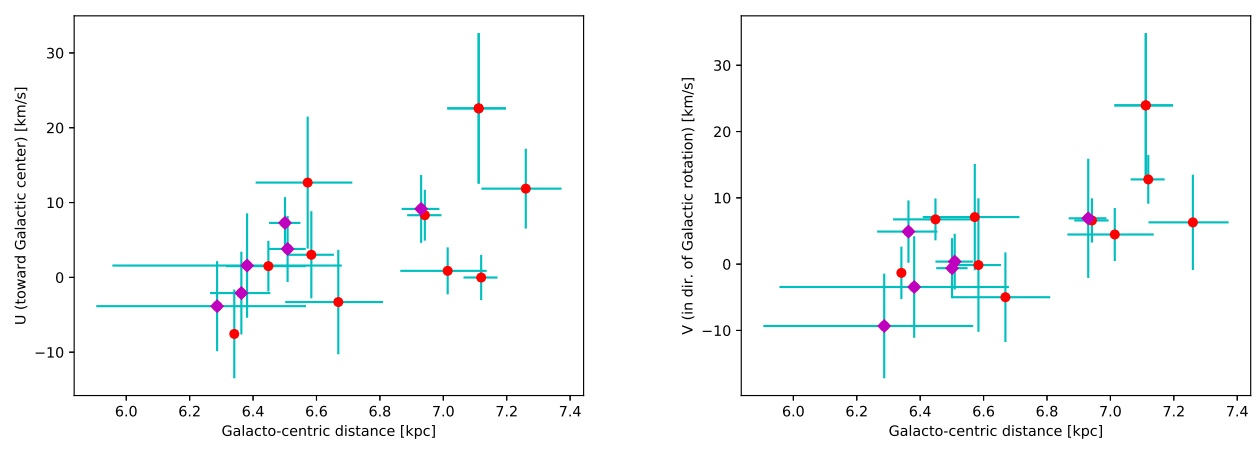

Figure 4: Left: Radial motion $(U)$ versus Galacto-centric distance, note that $U$ is positive when directed inward. Right: Azimuthal motion $(V)$ versus Galacto-centric distance, note that $V$ is positive when directed with Galactic rotation. Magenta diamonds mark values obtained in this work, red circles are literature values (see text for references).

Simulations have demonstrated the dynamical influence of the spiral arms quantified in welldefined patterns of peculiar velocity fields (see, for example, Grand et al. 2016). Grand et al. (2016) find that particles move radially outward (inward) and rotate backward (forward) on the trailing (leading) edge of the spiral arm. The combination of such a velocity field would then result in streaming motions along the spiral arm which consequently would permit radial migration across spiral arms. With our current sample of HMSFRs of Sgr Near, we find a similar signature in azimuthal motion, but we cannot constrain the radial motion. Future, larger samples of HMSFRs with VLBI astrometry might though provide a good observational testing ground for these theoretical predictions. 

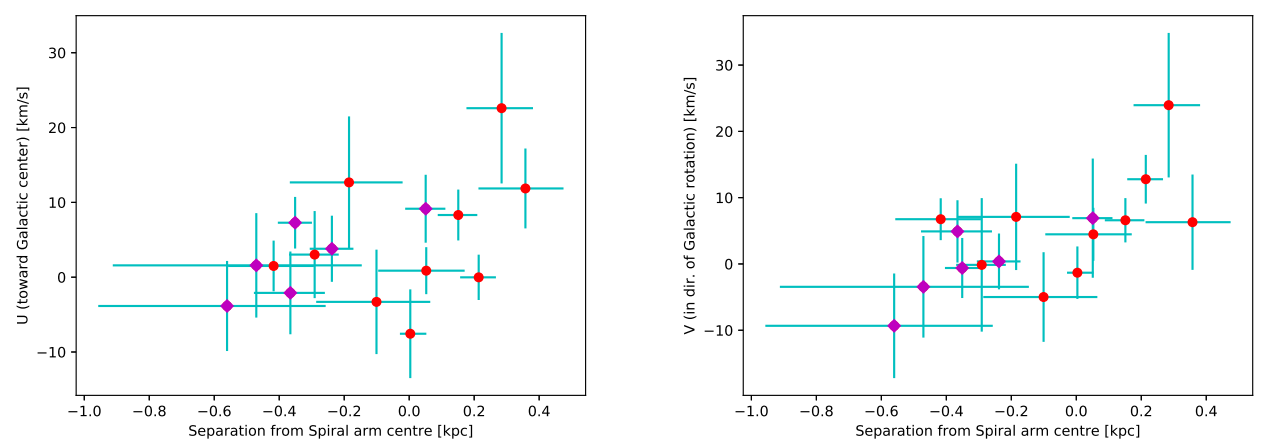

Figure 5: Left: Radial motion $(U)$ versus separation from spiral arm centre. Negative separations indicate positions inward of the spiral arm centre, positive outward. Right: Azimuthal motion $(V)$ versus distance from spiral arm centre. Magenta diamonds mark values obtained in this work, red circles are literature values (see text for references).

\section{Acknowledgements}

KLJR acknowledges funding from the Advanced European Network of E-infrastructures for Astronomy with the SKA (AENEAS) project, supported by the European Commission Framework Programme Horizon 2020 Research and Innovation action under grant agreement No. 731016. This presentation/publication was supported from the European Union's Horizon 2020 research and innovation programme under grant agreement No. 730562 [RadioNet].

\section{References}

Brunthaler, A., Reid, M. J., Menten, K. M., et al. 2011, Astronomische Nachrichten, 332, 461

Chibueze, J. O., Omodaka, T., Handa, T., et al. 2014, ApJ, 784, 114

Chibueze, J. O., Kamezaki, T., Omodaka, T., et al. 2016, MNRAS, 460, 1839

Dame, T. M., Hartmann, D., \& Thaddeus, P. 2001, ApJ, 547, 792

Grand, R. J. J., Springel, V., Kawata, D., et al. 2016, MNRAS, 460, L94

Kurayama, T., Nakagawa, A., Sawada-Satoh, S., et al. 2011, PASJ, 63, 513

Oh, C. S., Kobayashi, H., Honma, M., et al. 2010, PASJ, 62, 101

Reid, M. J., Menten, K. M., Brunthaler, A., et al. 2009a, ApJ, 693, 397

Reid, M. J., Menten, K. M., Brunthaler, A., et al. 2014, ApJ, 783, 130

Reid, M. J., Dame, T. M., Menten, K. M., \& Brunthaler, A. 2016, ApJ, 823, 77

Reid, M. J., Brunthaler, A., Menten, K. M., et al. 2017, AJ, 154, 63

Reid, M. J., Menten, K. M., Brunthaler, A., et al. 2019 in prep. 
Rygl, K. L. J., Wu, Y. W., Reid, M. J., et al. 2019 in prep.

Sato, M., Hirota, T., Reid, M. J., et al. 2010a, PASJ, 62, 287

Sato, M., Reid, M. J., Brunthaler, A., \& Menten, K. M. 2010b, ApJ, 720, 1055

Sato, M., Wu, Y. W., Immer, K., et al. 2014, ApJ, 793, 72

Schönrich, R., Binney, J., \& Dehnen, W. 2010, MNRAS, 403, 1829

Wu, Y. W., Sato, M., Reid, M. J., et al. 2014, A\&A, 566, A17

Wu, Y. W., Sakai, N., Reid, M. J., et al. 2019, ApJ, accepted, ArXiv:1901.09313

Xu, Y., Moscadelli, L., Reid, M. J., et al. 2011, ApJ, 733, 25

Zhang, B., Zheng, X. W., Reid, M. J., et al. 2009a, ApJ, 693, 419 